\title{
Complete mesocolic excision
}

\author{
L. Stocchi
}

Received: 29 May 2014/ Accepted: 29 May 2014/Published online: 4 July 2014

(C) Springer-Verlag Italia Srl 2014

The last 2 issues of Techniques in Coloproctology present 2 examples of the technique referred to as complete mesocolic excision (CME). Shin and colleagues report in the current issue on a series of 168 patients with stages II and III colon carcinoma treated with laparoscopic CME, who achieved a remarkable overall survival of $89.6 \%$ after a median follow-up of almost 5 years [1]. In their recently published experience, Storli and colleagues compared the results of resection for stage I-II colon carcinoma in 1 community hospital in which CME was used with results obtained in 2 control hospitals using standard surgical technique [2]. After a median follow-up of 50.2 months, the use of CME was associated with significantly improved 3 -year overall survival and disease-free survival, while the difference in cancer-specific survival was not statistically significant.

Both series, in different ways, pose the question of whether the technique of colonic dissection should be revisited to incorporate the principles of CME. The technique has been popularized by Hohenberger and colleagues who described the removal of colon cancer with ligation of vascular pedicles at their origin and preservation of the mesocolic investment of the removed specimen to minimize tumor spread. The principles of CME are intended to replicate in colon carcinoma the principles of total mesorectal excision, which have been associated with significant improvement of oncologic outcomes in rectal cancer and have been replicated in several centers throughout the world. In this respect, it is important to point out that CME

L. Stocchi $(\bowtie)$

Desk A30, Department of Colorectal Surgery, Digestive Disease Institute, Cleveland Clinic, 9500 Euclid Ave, Cleveland, OH 44195, USA

e-mail: stocchl@ccf.org requires a substantial technical dexterity when compared with standard technique, especially when using a laparoscopic approach. For example, a right colectomy with CME requires mobilization of the duodenum with the head of the pancreas and the mesenteric root to allow full exposure of the superior mesenteric vessels [3]. Pathological studies on the specimens resulting from such procedures have indicated advantages in surrogate metrics of oncologic benefits, namely preservation of the mesocolic envelope, increased lymph node retrieval and increased size of the removed specimen. However, the extent of the actual oncologic advantages deriving from CME remains unclear. For example, a study specifically assessing the impact of pathology grading of colon cancer surgical resection failed to demonstrate improved overall survival associated with mesocolic plane surgery, except for the subset of patients with stage III disease and did not report on cancer-specific survival [4]. On the other hand, the series, which did report improved cancer-specific outcomes after the introduction of CME when compared with an earlier time period, might not have adequately considered the influence on cancer outcomes of improved pathology staging and unequal use of chemotherapy treatments over time [3]. Storli and colleagues also reported a statistically marginal improvement in cancer-specific survival associated with CME as opposed to other important oncologic end points such as disease-free and overall survival. While lack of statistical power in the assessment of an individual end point is possible, an alternative explanation could be a difference in the comorbidity rate in the two groups, disproportionally accounting for the increased mortality noted in the standard group, as could be suggested by the $8.6 \%$ in-hospital mortality after standard colonic resections compared with $2.9 \%$ in the CME group. In the absence of reported data on comorbidity, this can only remain a 
speculation. Conversely, the study population of Shin and colleagues was remarkable for its patient fitness, with $94 \%$ of individuals whose American Society of Anesthesiologists score was I or II and more than $92 \%$ of stage III colon carcinomas treated with adjuvant FOLFOX, while the percentage of stage III patients receiving any chemotherapy in the United States is in the order of $50 \%$ [5].

An elegant dissection technique along embryological planes universally appeals to surgeons and is difficult to criticize. In this respect, there is little doubt that CME is an oncologically radical operation and the 2 series in question are no exception. CME might also have the advantage of improved staging accuracy, resulting in an increased identification of stage III patients and appropriate administration of chemotherapy with its inherent cancer benefits. This specific advantage could be very relevant, but remains difficult to demonstrate in retrospective case series such as that of Shin et al. or the comparative analysis of Storli and colleagues, who intentionally focused on stage I-II carcinomas.

While both groups should be commended for their attempt to optimize surgery for colon carcinoma, their contributions highlight once again the difficulty of objectively assessing the value of a particular surgical technique while adequately adjusting for the many covariates that independently influence oncologic outcomes.

Should additional data corroborate the routine use of CME as a novel standard of care, important practical consequences would come to the forefront. Firstly, as pointed out by Shin and colleagues., it is questionable whether a procedure of such technical precision, particularly when using a laparoscopic technique, is easily applicable to "typical Western patients," a tactful reference to the high rate of obesity in the United Stated and, to a lesser extent, in the rest of the Western world. Their single conversion in a patient population having a mean body mass index of $<24 \mathrm{~kg} / \mathrm{m}^{2}$ only reinforces such assumption.

In addition, as also alluded to by Storli et al., the potential adoption of $\mathrm{CME}$ would also raise the issue of adequate training requirements not only among colorectal surgeons but also, and most importantly, among general surgeons. For example, the vast majority of colon resections are performed in the United States by general surgeons rather than specialists [6]. Furthermore, the systematic adoption of all the principles of CME in the pathological evaluation of the surgical specimen, including the assessment of mesocolic integrity and morphometric measurements, would also require specific pathologist training and likely result in a significant increase in resource utilization.

While the present 2 series are intriguing in their suggestion that colon cancer treatment can benefit from optimization of surgical technique, future studies are necessary to definitely demonstrate the superiority of CME before we adopt it as the new standard of care.

Conflict of interest None.

\section{References}

1. Shin W, Amar AHY, Kim SH et al (2014) Complete mesocolic excision with D3 lymph node dissection in laparoscopic colectomy for stages II and III colon cancer: long-term oncologic outcomes in 168 patients. Tech Coloproctol. doi:10.1007/s10151-014-1134-z

2. Storli E, Søndenaa K, Furnes B et al (2014) Short term results of complete (D3) vs. standard (D2) mesenteric excision in colon cancer shows improved outcome of complete mesenteric excision in patients with TNM stages I-II. Tech Coloproctol 18:557-564

3. Hohenberger W, Weber K, Matzel K, Papadoupoulos T, Merkel S (2009) Standardized surgery for colon cancer: complete mesocolic excision and central ligation-technical notes and outcome. Colorectal Dis 11:354-365

4. West NP, Morris EJ, Rotimi O, Cairns A, Finan PJ, Quirke P (2008) Pathology grading of colon cancer surgical resection and its association with survival: a retrospective observational study. Lancet Oncol 9:857-865

5. Etzioni DA, El-Khoueiry B, Beart RW Jr (2008) Rates and predictors of chemotherapy use for stage III colorectal cancer. Cancer 113:3279-3289

6. Etzioni DA, Cannon RR, Madoff RD, Ault GT, Beart RW Jr (2010) Colorectal procedures: what proportion is performed by American Board of Colon and Rectal Surgery-Certified Surgeons? Dis Colon Rectum 53:713-720 